\title{
Study of blood microcirculation of pancreas in rats with alloxan diabetes by Laser Speckle Contrast Imaging
}

\author{
Polina A. Timoshina ${ }^{1 *}$, Alla B. Bucharskaya ${ }^{2}$, Denis A. Alexandrov ${ }^{2}$, Valery V. Tuchin ${ }^{1,3,4}$ \\ ${ }^{1}$ Research-Educational Institute of Optics and Biophotonics, Saratov National Research State University, Russia \\ ${ }^{2}$ Saratov State Medical University, Russia \\ ${ }^{3}$ Institute of Precision Mechanics and Control, Russian Academy of Sciences, Saratov, Russia \\ ${ }^{4}$ Interdisciplinary Laboratory of Biophotonics, National Research Tomsk State University, Russia \\ * e-mail: timoshina2906@mail.ru
}

\begin{abstract}
The blood microcirculation of the pancreas in rats with diabetes was studied using Laser Speckle Contrast Imaging (LSCI). The impact on blood flow of $\mathrm{x}$-ray contrast "Omnipaque $\mathrm{TM}_{-} 300$ " $(n=1.438)$ and aqueous solution of "Omnipaque ${ }^{\mathrm{TM}}-300$ " $(n=1.407)$ used as optical clearing agents (OCAs) was also investigated. The alloxan induced animal model of diabetes was exploited. The results obtained in the study of blood microcirculation disorders of pancreas in diabetes and under topical application of optical clearing agents show that disease development in animals causes changes in the microcirculatory system response to application of "Omnipaque ${ }^{\mathrm{TM}}-300$ " solutions. (C) 2017 Journal of Biomedical Photonics \& Engineering.
\end{abstract}

Keywords: speckle, blood flow, speckle contrast, diabet.

Paper \#3150 received 23 Jan 2017; revised manuscript received 10 Mar 2017; accepted for publication 13 Mar 2017; published online 29 Mar 2017. doi: 10.18287/JBPE17.03.020301. [Special Issue. Workshop "Biophotonics" of the XIII all-Russian Youth Samara conference-contest on optics and laser physics].

\section{References}

1. N. A. Palchikova, V. G. Selyatitskaya, and Y. P. Shorin, "A quantitative assessment of the sensitivity of the experimental animals to diabetogenic action of alloxan," Problems of Endocrinology, Russia, 65-68 (1987).

2. B. B. Tripathy (ed.), RSSDI: Textbook of Diabetes Mellitus, 2nd ed., Jaypee Brothers Medical Publishers, New Delhi (2012). ISBN: 9789350254899.

3. D. K. Tuchina, R. Shi, A. N. Bashkatov, E. A. Genina, D. Zhu, Q. Luo, and V. V. Tuchin, "Ex vivo optical measurements of glucose diffusion kinetics in native and diabetic mouse skin," Journal of Biophotonics 8(4), 332-346 (2015).

4. P. O. Bonetti, L. O. Lerman, and A. Lerman, "Endothelial dysfunction: a marker of atherosclerotic risk," Arterioscler. Thromb. Vasc. Biol. 23(2), 168-175 (2003).

5. V. V. Tuchin (ed.), Handbook of Optical Biomedical Diagnostics. Methods, 2nd ed., SPIE Press, Bellingham, USA (2016). ISBN: 9781628419122.

6. V. Doblhoff-Dier, L. Schmetterer, W. Vilser, G. Garhöfer, M. Gröschl, R. A. Leitgeb, and R. M. Werkmeister, "Measurement of the total retinal blood flow using dual beam Fourier-Domain Doppler Optical Coherence Tomography with orthogonal detection planes," Biomed. Opt. Express 5(2), 630-642 (2014).

7. Y. Huang, Z. Ibrahim, D. Tong, S. Zhu, Q. Mao, J. Pang, W. P. A. Lee, G. Brandacher, and J. U. Kang, "Microvascular anastomosis guidance and evaluation using real-time three-dimensional Fourier-domain Doppler optical coherence tomography," J. Biomed. Opt. 18(11), 111404 (2013).

8. Z. Chen, T. E. Milner, X. Wang, S. Srinivas, and J. S. Nelson, "Optical doppler tomography: Imaging in vivo blood flow dynamics following pharmacological intervention and photodynamic therapy," Photochemistry and Photobiology 67(1), 56-60 (1998).

9. J. D. Briers, "Laser Doppler, speckle and related techniques for blood perfusion mapping and imaging," Physiol. Meas. 22(4), 35-66 (2001).

10. K. Basak, M. Manjunatha, and P. K. Dutta, "Review of laser speckle-based analysis in medical imaging," Med. Biol. Eng. Comput. 50(6), 547-558 (2012). 
11. D. A. Zimnyakov, O. V. Ushakova, D. J. Briers, and V. V. Tuchin, Speckle Technologies for Monitoring and Imaging of Tissues and Tissue-Like Phantoms, 2nd ed., V. V. Tuchin (ed.), Handbook of Optical Biomedical Diagnostics, vol. 2: Methods, SPIE Press, Bellingham, USA (2016).

12. I. Sigal, R. Gad, A. M. Caravaca-Aguirre, Y. Atchia, D. B. Conkey, R. Piestun, and O. Levi, "Laser speckle contrast imaging with extended depth of field for in-vivo tissue imaging," Biomed. Opt. Express. 5(1), 123-134 (2014).

13. A. K. Dunn, H. Bolay, M. A. Moskowitz, and D. A. Boas, "Dynamic imaging of cerebral blood flow using laser speckle,” J. Cereb. Blood Flow Metab. 21, 195-201 (2001).

14. A. K. Dunn, "Laser speckle contrast imaging of cerebral blood flow," Ann. Biomed. Eng. 40(2), 367-377 (2012).

15. P. Li, S. Ni, L. Zhang, S. Zeng, and Q. Luo, "Imaging cerebral blood flow through the intact rat skull with temporal laser speckle imaging," Opt. Lett. 31(12), 1824-1826 (2006).

16. H. Cheng, Q. Luo, S. Zeng, S. Chen, J. Cen, and H. Gong, "Modified laser speckle imaging method with improved spatial resolution," J. Biomed. Opt. 8(3), 559-564 (2003).

17. X. Cheng, Y. Yan, and T. Q. Duong, "Laser speckle imaging of rat retinal blood flow with hybrid temporal and spatial analysis method," Proc. SPIE 7163, 716304 (2009).

18. R. Shi, M. Chen, V. V. Tuchin, and D. Zhu, "Accessing to arteriovenous blood flow dynamics response using combined laser speckle contrast imaging and skin optical clearing,” Biomed. Opt. Express, 6(6), 1977-1989 (2015).

19. A. I. Srienc, Z. L. Kurth-Nelson and E. A. Newman, "Imaging retinal blood flow with laser speckle flowmetry," Frontiers in Neuroenergetics 2(128), (2010).

20. H. Cheng, Q. Luo, S. Zeng, S. Chen, W. Luo, and H. Gong, "Hyperosmotic chemical agent's effect on in vivo cerebral blood flow revealed by laser speckle," Appl. Opt. 43(31), 5772-5777 (2004).

21. D. Zhu., K. Larin., Q. Luo, and V. Tuchin, "Recent progress in tissue optical clearing," Laser Photonics Rev. 7(5), 732-757 (2013).

22. Z. Wang, Q. Luo, H. Cheng, W. Luo, and Q. Lu, "Blood flow activation in rat somatosensory cortex under sciatic nerve stimulation revealed by laser speckle imaging,” Nat. Sci. 13(7), 522-527 (2003).

23. V. V. Tuchin, A. N. Bashkatov, E. A. Genina, V. I. Kochubey, V. V. Lychagov, S. A. Portnov, N. A. Trunina, D. R. Miller, S. Cho, H. Oh, B. Shim, M. Kim, J. Oh, H. Eum, Y. Ku, D. Kim, and Y. Yang, "Finger tissue model and blood perfused skin tissue phantom," Proc SPIE 7898, 78980Z (2011).

24. J. D. Briers, and S. Webster, "Laser speckle contrast analysis (LASCA): a non-scanning, full-field technique for monitoring capillary blood flow,” J. Biomed. Opt. 1(2), 174-179 (1996).

25. Y. Atchia, H. Levy, S. Dufour, and O. Levi, "Rapid multiexposure in vivo brain imaging system using vertical cavity surface emitting lasers as a light source," Appl. Opt. 52(7), 64-71 (2013).

26. R. Bonner, and R. Nossal, "Model for laser Doppler measurements of blood flow in tissue," Appl. Opt. 20(12), 2097-2107 (1981).

27. K. Khaksari, and S. J. Kirkpatrick, "Laser speckle contrast imaging is sensitive to advective flux," J.Biomed. Opt. 21(7), 076001 (2016).

28. K. Khaksari, and S. J. Kirkpatrick, "Combined effects of scattering and absorption on laser speckle contrast imaging," J. Biomed. Opt. 21(7), 076002 (2016).

29. D. Chen, J. Ren, Y. Wang, H. Zhao, B. Li, and Y. Gu., "Relationship between the blood perfusion values determined by laser speckle imaging and laser Doppler imaging in normal skin and port wine stains," Photodiagnosis and Photodynamic Therapy 13, 1-9 (2016).

30. A. Nadort, K. Kalkman, T. G. van Leeuwen, and D. J. Faber, "Quantitative blood flow velocity imaging using laser speckle flowmetry," Scientific Reports 6(1), 25258 (2016).

31. A. Bykov, T. Hautala, M. Kinnunen, and I. Meglinski, "Imaging of subchondral bone by optical coherence tomography upon optical clearing of articular cartilage," Journal of Biophotonics 9(3), 270-275 (2016).

32. A. Sdobnov, M. E. Darvin, J. Lademann, and V. Tuchin, "A comparative study of ex vivo skin optical clearing using two-photon microscopy,” J. Biophotonics, 1-9 (2017).

33. P. A. Timoshina, E. M. Zinchenko, D. K. Tuchina, M. M. Sagatova, O. V. Semyachkina-Glushkovskaya, and V. V. Tuchin, "Laser speckle contrast imaging of cerebral blood flow of newborn mice at optical clearing," Proc. of SPIE, to be published.

34. Omnipaque ${ }^{\mathrm{TM}}$ (2015).

35. M. P. Longinotti, J. A. T. González, and H. R. Corti, "Concentration and temperature dependence of the viscosity of polyol aqueous solutions," Cryobiology 69(1), 84-90 (2014). 


\section{Introduction}

Many diseases, such as cardio-vascular diseases, atherosclerosis, diabetes, chronic venous insufficiency, cause functional and morphological changes of blood flow in single vessels and blood microcirculation within the microvasculature. In the case of diabetes, diabetic angiopathy (generalized defeat of blood vessels) arises. There are two types of diabetic angiopathy: microangiopathy arising as result of destruction of small vessels - capillaries, arterioles, and venules, and macroangiopathy - ischemic brain disease, peripheral vascular occlusion - arising as result of destruction of large vessels [1]. Diabetes is characterized by the elevation of blood glucose level, for the reason that the peptide hormone insulin is produced insufficiently in the beta cells of the pancreas (type I insulin-dependent), or the body cells not responding properly to the insulin produced (Type II, non insulin-dependent or "adult onset diabetes") [2,3]. According to the data of World Health Organization, the incidence of diabetes in the world is about 347 million people. Diabetes is ranked as the third in the world after cardiovascular and oncological diseases. Hemodynamic changes in diabetes contribute to the emergence of hypoxemia in various organs, leading to retinopathy, neuropathy, nephropathy, coronary heart disease, and peripheral arterial disease. Thus, all these changes in blood flow and microcirculation within the microvasculature are primarily associated with endothelial dysfunction. The multiple functions of vascular endothelium include regulation of vessel integrity, vascular growth and remodeling, tissue growth and metabolism, immune responses, cell adhesion, angiogenesis, hemostasis and vascular permeability. Endothelial dysfunction is characterized by the following features: reduced endothelium-mediated vasorelaxation, hemodynamic deregulation, impaired fibrinolytic ability, enhanced turnover, overproduction of growth factors, increased expression of adhesion molecules and inflammatory genes, excessive generation of reactive oxygen species, increased oxidative stress, and enhanced permeability of the cell layer [4].

Currently, the most effective diagnostic methods for determining the physiological parameters of blood flow and microcirculation are the methods based on dynamic light scattering [5], as well as on principles of lowcoherence optical coherence tomography (OCT), the so called Doppler OCT (DOCT) [6-8]. One of the prospective methods for the assessment of blood flow is the Laser Speckle Contrast Imaging (LSCI) [9-15]. When a diffuse object is illuminated with laser light, a random interference known as a speckle pattern is produced. If there is movement within the object, the intensity of speckles fluctuates correspondingly. These fluctuations can be used to provide information about the red blood cells (RBCs) movement in vessels. A simple way of accessing this information is to image the speckle pattern - the fluctuations cause a blurring of the speckle pattern, leading to a reduction of the local speckle contrast [9]. Thus, the LSI is a noninvasive, contactless method that allows for visualization of capillary blood flow in a real-time without laser beam scanning. It is particularly attractive as an intra operative tool for measuring blood flow in ophthalmology, dermatology, neurosurgery and other fields of medicine, as it provides full-field relative flow speed maps with the use of only a coherent light source and a camera, both easily adaptable to conventional microscopes. Laser speckle contrast imaging (LSCI) techniques are based on the registration of spatial and temporal statistics of the speckle pattern, calculation of contrast of time-averaged dynamic speckles in dependence on the camera exposure time [9-16]. LSCI can be realized as Spatial (SLSCI) or Temporal (TLSCI) modifications. When SLSCI is used, contrast is calculated as the ratio of the standard deviation of the pixel intensity fluctuations to its mean value within selected area ( $\mathrm{N} \times \mathrm{N}$ pixels). The TLSCI is based on registration of temporal statistics and calculation of the image speckle contrast using a sequence of raw speckle images acquired along a few time points instead of a spatial window, i.e. contrast is calculated as the ratio of the standard deviation of the intensity fluctuations for a particular pixel at different times and mean intensity for this pixel $[15,16]$. The most useful modification appeared to combine these two techniques into one, called the Spatio-Temporal Laser Speckle Contrast Imaging (STLSCI). The contrast of the speckle image is calculated as the ratio of standard deviation of the intensity fluctuations for many pixels within a chosen area at different times and the pixel mean intensity averaged for all pixels of the area [17]. These techniques and some other LSI modifications, are often used in biomedical applications.

The applicability of the method is mainly determined by the imbedding depth of the blood vessels under study, being more accurate for superficial vessels. However the method of optical clearing, which reduces the efficiency of scattering from static scatterers, allows one to extend the applicability of the method to larger imbedding depths of the vessels. Combination of LSCI with immersion optical clearing demonstrates a high efficiency for evaluation of microhemodynamics of different tissues. For example, authors of Ref. [18] have quantified blood flow in arteries and veins in mouse skin due to a high quality of the speckle images. Also, LSCI was used in conjunction with confocal microscopy to monitor light-evoked changes in blood flow in retinal vessels [19]. This dual imaging technique permitted authors to stimulate retinal photoreceptors and measure vessel diameter with confocal microscopy while simultaneously monitoring blood flow with LSI. And the authors concluded that use of LSI to image retinal blood flow holds promise in elucidating the mechanisms mediating functional hyperemia in the retina and in characterizing changes in blood flow that occur during retinal pathology. Estimation of the impact of clearing agents on blood flow is of great importance for further application of this combined technology for more in depth imaging of blood hemodynamics. For 
example, Cheng et al. used LSCI to evaluate quantitatively OCA-induced changes of blood flow [20]. They found that after removing the rabbit skull and a small area of dura mater, the application of glycerol on the dura mater around the exposed cortex decreases the cerebral blood flow by $20-30 \%$ immediately. Zhu et al. [21] applied the LSCI to investigate both the shortterm and long-term effects of glycerol and glucose on blood vessels in chick chorioallantoic membrane (CAM).

In this study, we present results of blood flow measurements within the microvasculature of the pancreas in rats with diabetes and under influence of optical clearing agents by using Laser Speckle Contrast Imaging (LSCI). The influence of "Omnipaque ${ }^{\mathrm{TM}}-300$ " (radiopaque agent, active substance of iohexol) and of aqueous solution of "Omnipaque ${ }^{\mathrm{TM}}-300$ " was investigated.

\section{Methods and Materials}

\subsection{Laser speckle contrast imaging system}

The local estimation of the contrast $K$ for the fixed exposure time done within the areas with given number of speckles makes it possible to image tissue regions with essentially different velocity of scatterers $[9,10]$ :

$$
K_{k}=\sigma_{I k} / \bar{I}_{k}
$$

where $k$ is the number of frames in a sequence of speckle-modulated images, $\bar{I}_{k}$ and $\sigma_{I k}$ are the averaged over the analyzed frame scattered light intensity and the rms (root-mean-square) value of the fluctuation component of the pixel's brightness, respectively:

$$
\begin{aligned}
& \bar{I}_{k}=(1 / \mathrm{MN}) \sum_{m=1}^{M} \sum_{n=1}^{N} I_{k}(m, n), \\
& \sigma_{I k}=\sqrt{(1 / \mathrm{MN}) \sum_{m=1}^{M} \sum_{n=1}^{N}\left\{I_{k}(m, n)-\bar{I}_{k}\right\}^{2}},
\end{aligned}
$$

where $M$ and $N$ are the number of pixels in rows and columns of the analyzed area of the frame, respectively; $I_{k}(m, n)$ is the brightness of the $(m, n)$-pixel of the $k$ frame.

To perform the measurements and calculate contrast, we developed a software in the LabVIEW 8.5 environment (National Instruments, USA) that allows for real time recording of the intensity distribution of the speckle field with the rate of 100 frames per second. The monitoring of blood microcirculation in rats was carried out using a homemade experimental setup (Fig. 1). The observation area of the system was $1.5 \times 1.5$ $\mathrm{mm}^{2}$. Within this area, the spatial distribution of the speckle contrast was calculated from the measurements of intensity fluctuations by using Eqs. (1) - (3). The local contrast was quantified as the mean contrast within the small area of $7 \times 7$ pixels. Operator has a possibility to control field of view (FOV) of the optical system to provide measurements within a single vessel with directional blood flow. In the setup, He-Ne laser GN-5P
(Russia) was used as a light source $(\lambda=632.8 \mathrm{~nm})$. The monochrome CMOS camera (Basler A802f, number of pixels in the matrix $656 \times 491$, pixel size $9.9 \times 9.9 \mu \mathrm{m}, 8$ bit per pixel) with the fixed exposure time $\mathrm{T}$ of $10 \mathrm{~ms}$, combined with the LOMO objective $(10 \times$, St. Petersburg, Russia) was used as a detector. Figure 2 shows the spatial distribution of the measured local contrast (a) in coherent light, and its normalized distribution (b).

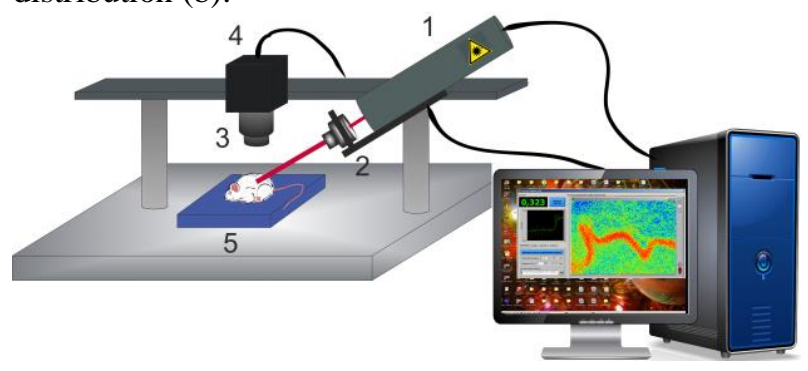

Fig. 1 1-He-Ne laser $(632.8 \mathrm{~nm}) ; 2$-objective (LOMO 20×); 3 microscope tube lens with objective (LOMO 10×); 4-CMOS-camera Basler A602f $(656 \times 491$ pxls, pxl size $9.9 \times 9.9 \mu \mathrm{m}, 8 \mathrm{bits} / \mathrm{pxl}) ; 5$ - rat under study.

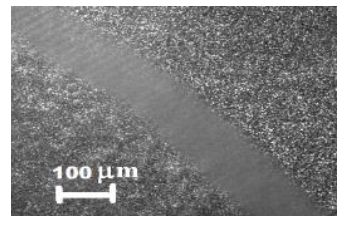

a)

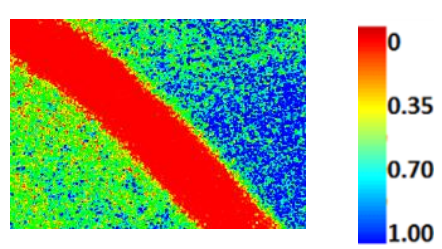

b)
Fig. 2 The images of a vessel (diameter $280 \mu \mathrm{m})$ : speckle image in laser light $(632.8 \mathrm{~nm})$ (a) and corresponding speckle contrast image as a calculated distribution of speckle contrast (1-0) (b).

The problem of quantitative velocity measurements is associated with understanding the inter-connection between the contrast of speckles and the velocity of scattering centers (or velocity distribution) [22]. As there is no an adequate theoretical description of this inter-connection, calibration should be used for the quantitative evaluation of the blood flow. This comparative study was conducted using a specially designed tissue phantom with capillaries (Fig. 3) [23]. The phantom had the following structure: the basic material was the epoxy resin with the inserted $\mathrm{TiO}_{2}(3$ $\mathrm{mg} / \mathrm{ml}$, diameter $200 \mathrm{~nm})$ and $\mathrm{SiO}_{2}(150 \mathrm{mg} / \mathrm{ml}$, diameter $1000 \mathrm{~nm}$ ) nanoparticles serving as scattering centers and Indian Ink $(0.2 \mu \mathrm{l} / \mathrm{ml})$ as an absorber; it was designed as a multi-layer plate of $27 \times 21 \times 3.2 \mathrm{~mm}$; thickness of the top layer (solid) was $150 \mu \mathrm{m}$; the second layer (porous) consisted of 29 capillaries with diameter of $180 \mu \mathrm{m}$; thickness of the third layer (solid) was $1 \mathrm{~mm}$; the fourth layer (porous) consisted of 29 capillaries with diameter of $180 \mu \mathrm{m}$; thickness of the fifth layer (solid) was $150 \mu \mathrm{m}$; the sixth layer (porous) consisted of 58 capillaries with diameter of $180 \mu \mathrm{m}$; thickness of the seventh layer (solid) was $1.54 \mathrm{~mm}$. Optical properties measured at $830 \mathrm{~nm}$ were similar to soft tissues with absorption coefficient of $0.48 \mathrm{~cm}^{-1}$, 
reduced scattering coefficient of $12.5 \mathrm{~cm}^{-1}$, and anisotropy factor of 0.88 . The flow of scattering fluid through this phantom modelling blood flow at specified velocities was provided by using a dispenser of drugs (MLW Lineomat, Germany).

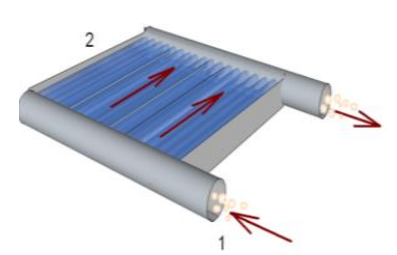

a)

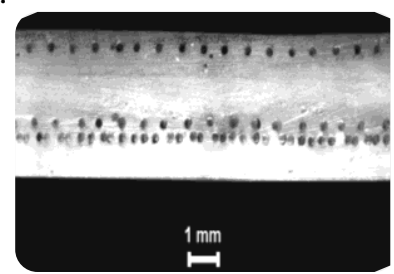

b)
Fig. 3 Phantom: schematic illustration: 1-suspension of particles (d 7-9 $\mu \mathrm{m}$ ), 2-phantom (a); image of phantom cross-section (b).

Under the assumption of purely ordered flow, the speckle contrast $K$ can be defined as follows [24-26]:

$$
\begin{aligned}
& K=\frac{\sigma_{s}}{\langle\boldsymbol{I}\rangle}= \\
& =\left[\frac{\tau_{c}}{2 T}\left\{\sqrt{2 \pi} \operatorname{erf}\left(\frac{\sqrt{2 T}}{\tau_{c}}\right)-\frac{\tau_{c}}{T}\left(1-\exp \left(-\frac{2 T}{\tau_{c}}\right)^{2}\right)\right]^{1 / 2}\right.
\end{aligned}
$$

where $\sigma_{s}$ is the standard deviation of the speckle intensity and $\langle I\rangle$ is the mean intensity, $T$ is the exposure time of the camera, $\tau_{c}$ is time of correlation. Again, it is worth noting that the above equation is in actuality a cumulative distribution function of a Gaussian probability distribution function, which is characteristic to directed flows.

The simplest approach leads to a characteristic velocity $v_{c}$ defined as follows [24-26]:

$$
v_{c}=\lambda / 2 \pi k \tau_{c}
$$

where $\lambda$ is the source wavelength, $k$ is the normalization factor which depends on the parameters of a Gaussian curve from Eq. (4), and the scattering properties of biological tissue or phantom. Calibration allowed us to determine the value of this coefficient as 0.24 , thus we can build a theoretical curve by using Eqs. (4) and (5) and compare it with the calibration curve received for the selected exposure time of $10 \mathrm{~ms}$ (Fig.4).

The theoretical curve fits well to the experimental calibration curve within velocity range from 0.1 to 2.5 $\mathrm{mm} / \mathrm{s}$ with the constant correction factor $k$. The presence of a correction factor was caused by multiple factors including the influence of static scatterers, random flow provided by upstream and downstream blood vessels, affecting value of the speckle contrast and thus causing reduction of accuracy of the flow rate estimates. These issues were discussed in the recently published papers [27-30].

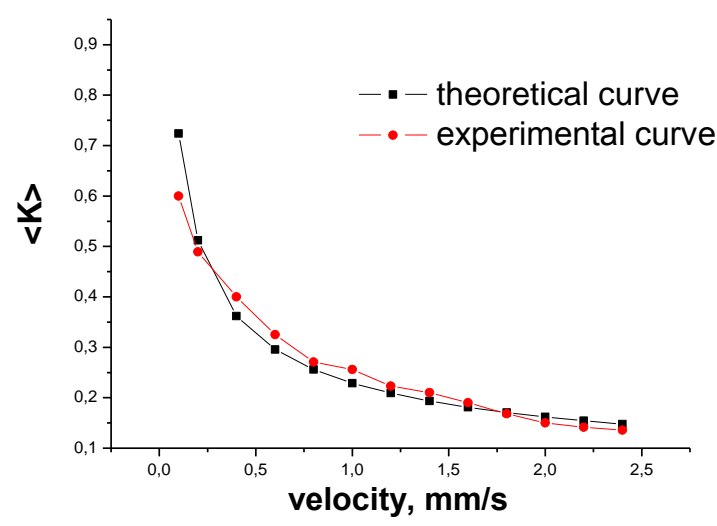

Fig. 4 Phantom studies with the selected exposure time of $10 \mathrm{~ms}$ : the relationship between speckle contrast and velocity of particles.

In this regard, we can introduce the concept of "reduced" velocity $v$ ' using Eqs. (4) and (5) to process phantom experimental data for contrast $K$ at the particular exposure time of the camera $T$ for the range of physiological velocities.

Reduced velocity $v^{\prime}$ can be associated with the velocity of blood flow determined from the speckle contrast $K$ measurements for the further assessment of blood circulation in in vivo studies. Results were presented as mean \pm standard error of the mean (SEM).

\subsection{Animal models}

We used 32 white laboratory rats weighing 300-500 g. Animals were divided into two groups: control (16 rats) and diabetic (16 rats). For modeling of diabetes in animals, we used alloxan-induced diabetes model [28, 29]. Experimental diabetes in rats was induced by a single subcutaneous injection of alloxan with a dose of $220 \mathrm{mg} / \mathrm{kg}$ body weight of the animal. Alloxan disturbed pancreas functioning leading to development of diabetes in rats [6]. Status of diabetes was confirmed by the increase of glucose level in the blood that was tested by using a commercial glucometer Accu-Chek Active (Roche Diagnostics, Germany). Average values of the glucose in the blood before the introduction of alloxan, and the day of the experiment (after 16 days) were $120 \pm 16,403 \pm 105 \mathrm{mg} / \mathrm{dl}$, respectively. We can assume that this is an initial stage of diabetes.

Under general anesthesia solution Zoletil $0.2 \mathrm{~mL}$, laparoscopy was performed. Then the changes of blood flow were evaluated.

All procedures with animals were performed in strict accordance with "Rules for Conducting Qualitative Clinical Trials in the Russian Federation" (approved by the Ministry of Health of the Russian Federation and enacted on January 1, 1999), appendix 3 to Order No. 755 of the Ministry of Health of the USSR of 10.08.77, the provisions of WMA Declaration of Helsinki (2000) and the recommendations contained in the European Community Directives (No. 86 / 609EC).

In addition, vascular permeability studies were performed by applying optical clearing agent 
"Omnipaque ${ }^{\mathrm{TM}}-300 "$ (a radiopaque agent for $\mathrm{x}$-ray image contrasting with the active substance iohexol) and a dilute aqueous solution of "Omnipaque ${ }^{\mathrm{TM}}-300$ " (7ml "Omnipaque ${ }^{\mathrm{TM}}-300 ", 3 \mathrm{ml}$ of distilled water), namely, the analysis of its effects on blood flow in pancreatic vessels. The solutions were applied to the tissue site topically using a pipette in a volume of 0.5 $\mathrm{ml}$.

The Omnipaque ${ }^{\mathrm{TM}}$ is currently not yet widely used for tissue optical clearing, however a few impressive applications are already described, i.e., for subcartilaginous bone imaging by OCT [31], for clearing of ex vivo pig skin at two-photon microscopy [32], and to improve images of cerebral blood flow in newborn mice [33]. The major attractiveness of the agent is that it is a non-ionic X-ray contrast iodinebased solution that due to low osmolarity comparable with osmolarity of blood plasma and cerebrospinal fluid (CSF) does not cause tissue damage at prolonged topical application or intra-tissue injection [34].

\section{Results and discussion}

Results of the measurement of "reduced" velocity $v$ ' of blood flow in vessels of pancreas for control and diabetic groups of laboratory rats are shown in Fig. 5.

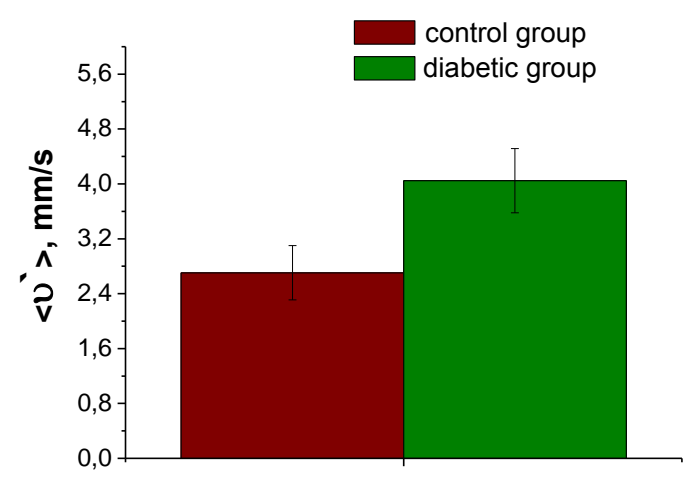

Fig. 5 Changes of blood flow in vessels of pancreas under alloxan diabetes, the difference with control is significant $(p<0.5)$.

The development of diabetes affected the change of blood flow relative to the control group of animals, the difference is significant ( $\mathrm{p}<0.5)$. From Fig. 5 it follows that the flow velocity in diabetic rats was $40 \%$ above the velocity of the control group.

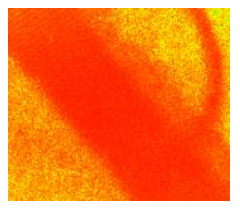

a)

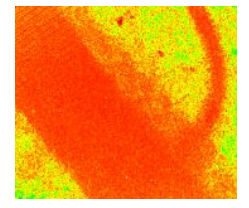

b)

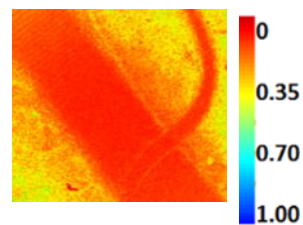

c)
Fig. 6 Contrast images of blood vessels of pancreas after using aqueous solution of $70 \%$ "Omnipaque ${ }^{\mathrm{TM}}-300$ ": a) without influence, b) after $1 \mathrm{~min}$, c) $5 \mathrm{~min}$.
Figure 6 shows the distribution of contrast of the speckle images when pancreas was subjected topically to an aqueous $70 \%$ solution of "Omnipaque ${ }^{\mathrm{TM}}-300$ ". This is evident that use of the OCA improved the speckle contrast images of studied vessels to $5^{\text {th }}$ min of its application.

Further, in Fig. 7 the normalized curves of changes of pancreatic blood flow of control and diabetic groups of laboratory animals under the influence of $100 \%$ "Omnipaque $\mathrm{TM}_{-}-300 "$ and aqueous solution of $70 \%$ "Omnipaque ${ }^{\mathrm{TM}}-300 "$. Data were averaged over 8 laboratory animals for each group and for each solution, respectively.

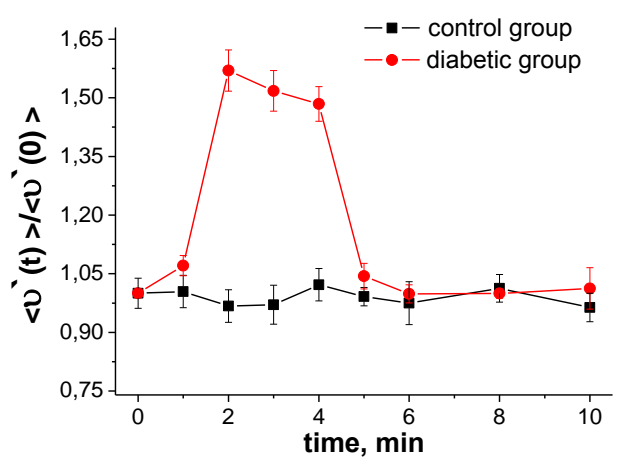

(a)

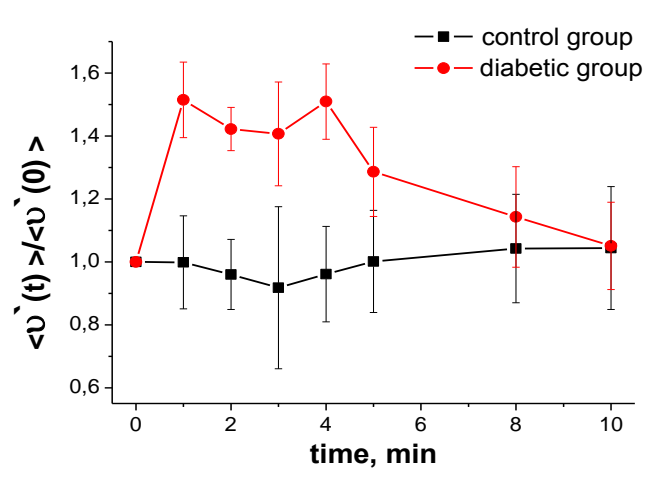

b)

Fig. 7 Blood flow in the pancreas of rats in both groups exposed to $100 \%$ "Omnipaque $\mathrm{TM}^{\mathrm{TM}}-300$ ” (a) and 70\% “Omnipaque ${ }^{\mathrm{TM}}-300$ ” (b).

Application of $100 \%$ "Omnipaque ${ }^{\mathrm{TM}}-300$ " demonstrates $65 \%$-increase of blood flow as $70 \%$ "Omnipaque ${ }^{\mathrm{TM}}-300 "$ gives $50 \%$-increase of blood flow in the group of diabetic animals. In both cases to $10^{\text {th }}$ min, blood flow velocity was completely restored. Blood flow in the control group did not show any noticeable changes. Increased blood flow after application of optical clearing agent could be caused by increased vascular endothelial permeability at diabetes even in early stages of disease. It was also found that the diameter of the vessel under the influence of the solutions did not change. Based on these results, it is worth noting that "Omnipaque ${ }^{\mathrm{TM}}-300$ " causes some changes in the blood flow in the pancreatic vessels only in diabetic mice, and this fact should be taken into 
account in the diagnostic applications of the combined technology that is proposed in this study. Thus, application of "Omnipaque ${ }^{\mathrm{TM}}-300$ " could be used not only for getting of better speckle images of blood flow distribution in the living organ at surgical procedures, but also for monitoring of some complications related to increased vascular endothelial permeability like at diabetes.

The assessment of the effect of the agent on blood flow by LSCI opens very urgent perspectives to combine blood circulation measurements with tissue optical clearing in the course of transplantation. It is important to note that practically all OCAs are cryogenic agents used for keeping living organs before transplantation [35].

\section{Conclusion}

The calibration method of speckle contrast imaging using a phantom simulating directed blood circulation in the biological tissue, allows one to calculate the spatial distribution of the contrast and evaluate the dependence of contrast speckle - image relative velocity of RBC. This allows one to move from a qualitative analysis of blood flow alterations to the quantitative, namely the "reduced" velocity $v_{c}^{\prime}$ determined with the help of phantom calibration. Due to phantom calibration laser speckle contrast imaging may be useful for quantitative monitoring of microhemodynamics of pancreas and other organs and study of vascular endothelial permeability at diabetes.

The ability of LSCI to measure blood flow velocity in a real time is prospective feature to be used in transplantation technologies and in emergency surgery to assess the state of internal organs and microhemodynamic pathologies. Also, one of the prospective applications of LSCI technique is a noninvasive monitoring of cerebral blood flow in the brain of small animals without craniotomy under conditions of immersion of optical clearing of the scalp and cranial tissues by means of hyperosmotic agents.

\section{Acknowledgments}

The presented results were made possible with support received from the grants of Russian Ministry of Education and Science No. 3.1586.2017/PCh, Russian Presidential grant NSh-7898.2016.2, and the Government of the Russian Federation grant 14.Z50.31.0004.

The authors express their gratitude to O. V. Semyachkina-Glushkovskaya for help in choosing of a model of diabetes in animals and to V. V. Lychagov for designing and manufacturing of tissue phantom. 